\title{
Benefit of Pulmonary Subspecialty Care for Children with Sickle Cell Disease and Asthma
}

\author{
Shikha Saxena ${ }^{1}$, Olufunke Afolabi-Brown ${ }^{2}$, Lance Ballester ${ }^{2}$, Nathaniel Schmucker ${ }^{2}$, Kim \\ Smith-Whitley ${ }^{2}$, Julian Allen ${ }^{2}$, and Anita Bhandari² \\ ${ }^{1}$ Monroe Carell Junior Children's Hospital at Vanderbilt \\ ${ }^{2}$ The Children's Hospital of Philadelphia
}

May 29, 2021

\begin{abstract}
Objective: Asthma is a recognized comorbidity in children with sickle cell disease (SCD). It increases risk of acute chest syndrome (ACS), vaso-occlusive episodes, and early mortality. We aim to determine whether evaluation and management of children with sickle cell disease (SCD) and asthma by a pulmonologist reduces rate of asthma exacerbation and ACS. Methods: The study included 192 patients with SCD (0-21 years) followed at Children's Hospital of Philadelphia Hematology between 01/01/2015 and 12/31/2018 with a diagnosis of asthma, wheeze, or cough. Patients were placed in two groups: those evaluated by a pulmonologist (SCD-A-P) and those not (SCD-A). Rates of emergency department (ED) visits and hospitalizations for asthma exacerbation and ACS were compared between groups and before/after initial pulmonology visit. Results: SCD-A-P $(\mathrm{n}=70)$ had lower baseline pulmonary function, hemoglobin, and hematocrit compared to SCD-A ( $\mathrm{n}=122)$. SCD-A-P had a higher average rate of hospital visits for asthma exacerbation and ACS per year compared to SCD-A: 0.84 [0.56-1.12] versus 0.31 [0.18-0.43], $(\mathrm{p}<0.001)$. For SCD-A-P patients with at least one hospital visit $(\mathrm{n}=48)$, the average rate decreased from 3.93 [1.57-6.29] before initial pulmonary visit to 0.85 [0.48-1.23] after the visit $(\mathrm{p}=0.014)$. Conclusion: SCD-A-P had more severe baseline disease and higher rates of ED visits and hospitalizations for asthma exacerbation and ACS compared to SCD-A, but the rates significantly decreased following pulmonology consultation. These findings support the pulmonologist's role in the multidisciplinary care of SCD patients. Further studies are needed to help establish evidence-based asthma guidelines for children with SCD.
\end{abstract}

\section{Introduction}

Sickle cell disease (SCD) affects about 100,000 people in the U.S ${ }^{1}$. The prevalence of pulmonary disease in children with SCD ranges from 20 to 48 percent ${ }^{1,2}$. Pulmonary complications can accelerate morbidity and mortality, and children with SCD can suffer from a shortened lifespan by two to three decades ${ }^{1,3,4}$.

Several studies have demonstrated that children with SCD are more likely to have abnormal lung function, including airway hyperreactivity, lower airway obstruction, and restrictive physiology, compared to children without SCD 5,6,7,8. Lung function impairment can be detected early in life; infants with SCD have been found to have lower expiratory flows compared to healthy controls ${ }^{7}$. More specifically, patients with SCD are more likely to have asthma than patients without SCD ${ }^{2}$. The pathophysiology is thought to be due to overlapping inflammatory mechanisms in asthma and SCD. Studies performed in the mouse model have demonstrated an increased inflammatory response to allergic triggers in the lungs of SCD mice compared to in the lungs of wild type mice ${ }^{9,10}$. Hemolysis associated with SCD has been hypothesized to induce inflammation in the lungs, and bronchoconstriction and inflammation associated with asthma results in a regional hypoxemia and worsens erythrocyte sickling. This sets the stage for a cycle of inflammation and infarction ${ }^{11,12,13}$. 
Asthma in children with SCD is associated with an increased incidence of acute chest syndrome (ACS) $14,15,16$, vaso-occlusive pain crises ${ }^{14,17}$, pulmonary hypertension ${ }^{12}$, and stroke ${ }^{12,15}$. The Cooperative Study for SCD found that children with SCD and asthma had nearly twice as many episodes of ACS compared to non-asthmatic children with SCD. These patients also developed ACS at an earlier age ${ }^{17}$. A multinational study found that asthma is a significant risk factor for emergency department visits for pain crises and ACS in children with SCD ${ }^{18}$. Furthermore, asthma ${ }^{19}$ and ACS ${ }^{4}$ were associated with early death in longitudinal studies including children and adults with SCD.

Optimizing asthma management in SCD is essential in reducing morbidity for children with this lifelong disease, and early recognition and prevention of asthma may ultimately improve quality of life and survival in children with SCD ${ }^{20}$. While patients with asthma can be cared for by the general pediatrician, they may require referral to a pulmonologist if they are high risk, described as requiring emergency department (ED) visits or admission, having persistent poor lung function despite treatment, or having complicated asthma ${ }^{21}$. Pediatric pulmonary consultation has been shown to decrease asthma hospitalizations in otherwise healthy children $^{22}$. In the SCD population, an integrated care model involving providers with specialized experience in asthma resulted in enhanced evidence-based asthma care ${ }^{23}$. However, there are no clear guidelines regarding when patients with SCD and asthma should be referred to the pulmonologist and no clear evidence whether this referral improves outcomes.

This study aimed to determine whether the evaluation and management of pediatric patients with SCD and asthma by a pulmonologist reduced their rate of asthma exacerbations and ACS requiring ED visits and hospitalization.

\section{Methods}

\section{Study population}

A single-center retrospective cohort study was performed involving patients between ages 0-21 years with SCD and asthma, cough, or wheeze who were followed by the Children's Hospital of Philadelphia (CHOP) Division of Hematology between January 1, 2015 and December 31, 2018. Patients were included if they had at least two outpatient visits completed with Hematology within this timeframe to exclude patients who had only been seen at CHOP once for a second opinion. Convenience sample was obtained of all available patients who met the inclusion criteria.

\section{Data collection and study design}

The study was conducted using chart review of existing electronic medical records under CHOP Institutional Review Board approval. Patients were identified by querying the electronic medical record for ICD-9 and ICD-10 diagnosis codes for SCD, asthma, wheeze, and cough within the date and age ranges specified above. Data were collected on patient demographics, past medical history, prescribed medications and therapies, laboratory results, pulmonary function tests (PFTs), asthma control test (ACT), asthma care plan (ACP), outpatient hematology and pulmonary visits, and ED visits and admissions.

Patients were divided into two groups: those who had at least one outpatient evaluation at CHOP Division of Pulmonary and Sleep Medicine within the study period (group SCD-A-P) and those who did not (group SCD-A). The primary outcome was the rate of ED visits and inpatient admissions for asthma exacerbation, albuterol-responsive cough/wheeze, and ACS. The diagnoses for the hospital visits were based on ICD-9 and ICD-10 codes as well as physician documentation. Average rates of ED visits and admissions for these diagnoses were compared between groups SCD-A-P and SCD-A. The SCD-A-P patients with at least one hospital visit had their average rates of hospital visits compared before and after the initial pulmonary visit.

\section{Statistical analysis}

The rate of ED visits and inpatient admissions for asthma exacerbation and ACS for each patient was calculated by dividing the number of events by the patient's follow up time (in years) in the study. The 
exact length of follow up between January 1, 2015 and December 31, 2018 was determined by the time between the first and last hematology outpatient visit, pulmonary outpatient visit, or hospital visit for asthma exacerbation or ACS, whichever duration was longest.

T-tests were performed to compare continuous variables, including the average rates of ED visits and admissions, between the two groups. Chi-squared tests were used to compare categorical variables, including the baseline characteristics, between the two groups. For patients in group SCD-A-P with at least one hospital visit, rates before and after the initial pulmonary visit were compared using a paired t-test, spaghetti plots, and modeling using a Poisson-distributed generalized estimating equation with exchangeable covariance assumed between the two time points of the same patient. The statistical analyses were performed and plotted using Stata, SAS, and R.

\section{Results}

\section{Patient characteristics}

A total of 192 patients with SCD and asthma, cough, or wheeze were identified; 70 patients were followed by a pulmonologist (SCD-A-P) and 122 patients were not (SCD-A). The overall mean number of outpatient pulmonary visits during the study period for patients in SCD-A-P was 3.14 [2.56-3.72]. Demographic and clinical characteristics are reported in Table 1 . The mean follow up time for the cohort was 2.88 years and was comparable between the two groups (2.89 years for SCD-A-P and 2.88 years for SCD-A, p=0.95). Patients in SCD-A-P were slightly younger than in SCD-A (mean 9.8 versus 11.5 years, $\mathrm{p}=0.04$ ). Two patients were deceased by the end of the study period (one patient in the SCD-A-P group for respiratory failure associated with cardiac dysfunction, and one patient in the SCD-A group for respiratory failure related to osteomyelitis).

SCD type SS (62\%) was the most common genotype, followed by SCD type SC (27\%). Type SS was more prevalent in SCD-A-P $(74 \%)$ than in SCD-A $(55 \%)(\mathrm{p}=0.008)$, while type SC was more prevalent in SCD-A $(33 \%)$ than in SCD-A-P (16\%) ( $\mathrm{p}=0.01)$. Regarding asthma severity, $41 \%$ of patients in SCD-A-P had mild persistent asthma versus $25 \%$ in SCD-A $(\mathrm{p}=0.015)$, and $21 \%$ of patients in SCD-A-P had moderate persistent asthma versus $8 \%$ in SCD-A ( $\mathrm{p}=0.009)$. However, $31 \%$ of patients in SCD-A had unspecified asthma severity versus $6 \%$ in group SCD-A-P $(\mathrm{p}<0.001)$. There were no patients in the severe persistent category. There was no significant difference in the percentage of patients with a past episode of ACS between the two groups. Patients in SCD-A-P had a higher rate of sleep disordered breathing including obstructive sleep apnea (OSA) $(\mathrm{p}=0.018)$, and they were more likely to be prescribed home supplemental oxygen or non-invasive positive pressure ventilation $(\mathrm{p}<0.001)$ compared to patients in SCD-A.

Patients in SCD-A-P were more likely than patients in SCD-A to be prescribed short acting beta agonists (albuterol or levalbuterol) $(\mathrm{p}=0.004)$, preventative asthma medications (inhaled corticosteroid, combination inhaled corticosteroid and long acting beta agonist, or montelukast) $(\mathrm{p}<0.001)$, and hydroxyurea $(\mathrm{p}=0.008)$. Patients in SCD-A-P had higher rates of completion for ACT, ACP, and PFTs $(\mathrm{p}<0.001)$. On initial PFTs, patients in SCD-A-P had lower forced expiratory volume in 1 second $\left(\mathrm{FEV}_{1}\right)(\mathrm{p}=0.04)$ and lower ratio of $\mathrm{FEV}_{1}$ to forced vital capacity $\left(\mathrm{FEV}_{1} / \mathrm{FVC}\right)(\mathrm{p}<0.001)$. Patients in SCD-A-P also had lower initial hemoglobin $(\mathrm{p}=0.02)$ and hematocrit $(\mathrm{p}=0.01)$.

Comparison in hospital visits between $S C D-A-P$ and $S C D-A$

Table 2 compares data on the hospital visits for asthma exacerbation and ACS between the two groups during the entire study period. Of the cohort of 192 patients, 96 patients (50\%) had at least one hospital visit, with 48 of these patients in SCD-A-P and 48 in SCD-A. There was a significantly higher proportion of patients in SCD-A-P than SCD-A with at least one hospital visit for asthma $(\mathrm{p}=0.007)$ and ACS $(\mathrm{p}<0.001)$. Patients in SCD-A-P also had an average of 1.94 [1.45-2.44] hospital visits per person and an average of 0.84 [0.56-1.12] hospital visits per year compared to patients in SCD-A with an average of 0.71 [0.51-0.92] hospital 
visits per person $(\mathrm{p}<0.001)$ and an average of $0.31[0.18-0.43]$ hospital visits per year $(\mathrm{p}<0.001)$. Of the 96 patients with hospital visits, $43 \%$ required respiratory support, $29 \%$ required simple blood transfusion, and $5 \%$ required admission to the intensive care unit (ICU), without any significant difference between the two groups.

\section{Comparison in hospital visits before and after pulmonary visit}

Of the 70 patients in SCD-A-P, $48(69 \%)$ had at least one ED visit or admission for asthma exacerbation, albuterol-responsive cough/wheeze, or ACS. The average rate of hospital visits per year for these patients decreased from 3.93 [1.57-6.29] before the initial pulmonary visit to 0.85 [0.48-1.23] after the initial pulmonary visit $(\mathrm{p}=0.014)$. The mean follow up time within the study was 1.00 years before the initial pulmonary visit and 1.83 years after the pulmonary visit. The figure depicts the change in rates of hospital visits for individual patients, before and after the initial pulmonary visit. The figure includes $42 \mathrm{SCD}-\mathrm{A}-\mathrm{P}$ patients with at least one hospital visit ( 6 out of 48 patients were excluded because their initial pulmonary visit was either the first or last date in the study period, making it difficult to determine the number of hospital visits before or after that pulmonary visit, respectively). In particular, the 10 patients with the highest rates prior to the pulmonary visit experienced decreases in rates after the pulmonary visit.

Rate ratios were calculated for the patients in SCD-A-P with at least one ED visit or admission. The rate ratio from pre- to post- pulmonary visit was $0.40[0.27,0.58](\mathrm{p}<0.001)$, indicating the rate of hospital visits after the pulmonary visit was 0.40 times the rate before the pulmonary visit. The rate ratio after adjusting for additional covariates of age, sex, SCD type, asthma severity, medications, and past medical history (ACS, OSA, pulmonary hypertension) was similar at $0.44[0.30,0.64](\mathrm{p}<0.001)$.

\section{Discussion}

Asthma is a common coexisting condition in children with SCD and is associated with significant morbidity. Studies show that asthma increases the risk of complications including vaso-occlusive pain crises, stroke, ACS, and early mortality. Early detection and treatment of asthma are key in preventing such complications. While studies have established the benefit of pulmonary referral for otherwise healthy patients with asthma, this study explores the potential benefit in patients with SCD and asthma.

In this study, patients with SCD and asthma who were referred to a pulmonologist had more severe underlying disease and thus higher rates of hospital visits for asthma exacerbation and ACS at baseline compared to patients who were not referred to a pulmonologist. Asthma has been previously shown to be associated with an increased frequency of ED visits for ACS in patients with SCD ${ }^{18}$. Once under care of the pulmonologist, patients in our study had a significant decrease in the rate of hospital visits for asthma exacerbation and ACS.

The SCD-A-P group in our study had lower initial $\mathrm{FEV}_{1} / \mathrm{FVC}$ and ultimately higher rates of ACS, suggesting more severe lung disease at baseline. There have been variable associations of PFTs with ACS reported in the literature. One study demonstrated that SCD patients with a history of ACS had significantly lower $\mathrm{FEV}_{1} / \mathrm{FVC}$ ratio and forced expiratory flow at $25-75 \%$ of pulmonary volume $\left(\mathrm{FEF}_{25-75}\right)$ compared to SCD patients without a history of ACS ${ }^{24}$. Another study similarly found that SCD patients with decreased $\mathrm{FEV}_{1} / \mathrm{FVC}$ ratio had higher rates of hospitalizations for pain and ACS ${ }^{25}$. However, a more recent study which enrolled SCD patients regardless of presence or absence of asthma demonstrated that lung function as measured by spirometry and plethysmography was not correlated with past or future pain or ACS episodes 26. In our study, only patients with SCD and asthma were included. There was no significant difference in history of ACS between the SCD-A-P and SCD-A groups, but the SCD-A-P group with lower average baseline PFTs experienced higher rates of ACS during the study, suggesting that patients with lower baseline PFTs should be considered for referral to the pulmonologist.

Regarding asthma severity, the most common classification was mild persistent asthma for SCD-A-P patients and mild intermittent asthma for SCD-A patients. However, $31 \%$ of SCD-A patients were unspecified, 
suggesting that the pulmonologist may be more inclined to characterize asthma severity.

The majority of patients in the SCD-A-P group had the homozygous genotype of SCD type SS, and this group had higher rates of hospital visits for asthma and ACS compared to group SCD-A. Patients with this genotype are known to have more severe disease than patients with the heterozygous genotype (SCD type SC) ${ }^{1,15}$, and they are at higher risk for ACS compared to other SCD genotypes ${ }^{27}$. Thus, patients with SCD type SS should perhaps be referred to the pulmonologist more readily compared to patients with other genotypes.

A higher proportion of patients in the SCD-A-P group also had a diagnosis of OSA and were prescribed home respiratory support. Patients with OSA and SCD have been shown to have more severe nocturnal desaturation and hypercapnia compared to patients with OSA alone, and nocturnal hypoxemia can predispose to erythrocyte sickling ${ }^{28}$. Thus, we speculate that the patients in our study with OSA may be at higher risk for complications including ACS, although the numbers are too low to confirm this association.

In our study, patients referred to a pulmonologist had lower initial hemoglobin and hematocrit levels. In addition, they were more likely to have been prescribed hydroxyurea at some point during the study period. Hydroxyurea increases the fetal hemoglobin level, which has been correlated with longer life expectancy ${ }^{4}$, and it also increases total hemoglobin level, reduces frequency of pain crises and ACS, and reduces need for blood transfusions ${ }^{1}$. Past studies have shown that patients with asthma and SCD require more blood transfusions than patients with SCD alone ${ }^{15,17}$. In our study, $29 \%$ of patients with at least one ED visit or admission required a blood transfusion. Taken together, these data suggest a potential role for hydroxyurea in the treatment of SCD patients with asthma.

Patients treated by a pulmonologist were more likely to be prescribed asthma medications including a shortacting bronchodilator, an inhaled corticosteroid, a combination inhaled corticosteroid and long acting beta agonist, and montelukast. Published recommendations from experts suggest treating acute wheeze in SCD patients with a short-acting beta agonist, short-term course of systemic corticosteroids, and supplemental oxygen (if hypoxemic). Long term, patients may be placed on an inhaled corticosteroid or leukotriene receptor antagonist ${ }^{11,12,20,29}$. The physiologic benefit of albuterol has been demonstrated in the literature, as patients with SCD have higher rates of asthma, airway hyperreactivity, and bronchodilator responsiveness $8,2,16,30$. Inhaled corticosteroids are another mainstay of asthma treatment, and otherwise healthy children with asthma are more likely to have a controller prescription after pediatric pulmonary consultation ${ }^{22}$, but not many studies have been performed in the SCD population. Leonard et al showed that inhaled corticosteroids did not have an impact on ACS morbidity, but the study included SCD patients with and without asthma ${ }^{31}$. Evidence on the use of systemic corticosteroids is also mixed due to adverse effects such as pain crises in the SCD population. Steroids have been associated with increased lengths of stay and readmission rates when used for treatment of $\mathrm{ACS}^{32,33}$. Prospective clinical trials are needed to establish evidence-based guidelines on the use of asthma medications in patients with SCD.

Our study emphasizes the severity of respiratory illness for patients with SCD and asthma, with $43 \%$ of admitted patients requiring respiratory support and $5 \%$ requiring ICU-level care. This further highlights the need for research and evidence-based asthma guidelines for patients with SCD.

There is a paucity of literature exploring the role of the pulmonologist in this population. A quality improvement study performed at an integrated SCD and asthma clinic at a community health center demonstrated improved adherence to evidence-based asthma guidelines and decreased lower airway obstruction after implementation of the integrated model. While ED and hospital encounters for asthma and ACS decreased, the change was not statistically significant. The authors propose that this lack of statistical significance was related to no validated asthma guidelines for children with SCD ${ }^{23}$.

In our study, patients who were followed by the pulmonologist were more likely to receive standardized asthma care, including classification of asthma severity, PFTs (despite their younger age), ACTs, ACPs, and asthma medications. Furthermore, the patients experienced a significant decline in rate of hospital visits for asthma and ACS following evaluation by a pulmonologist. It is unclear which of the pulmonologist's 
interventions had the greatest impact, and this should be investigated in future studies. Still, these findings point to a clear benefit of pulmonary subspecialty care in the overall management of children with SCD and asthma, particularly for patients with SCD type SS and lower baseline lung function and hemoglobin levels.

Unfortunately, there are inequalities in SCD funding and clinical care in the United States, which may contribute to inadequate evidence-based guidelines and perpetuate health care disparities. Federal and foundational funding for research is lower for SCD than for cystic fibrosis (CF), despite both being severe progressive genetic disorders ${ }^{34}$. There is also an inappropriate delay in the implementation of clinical advancements in SCD which negatively impacts quality of care ${ }^{35}$. For patients with SCD, multidisciplinary specialty clinics are not as available, networked, or widely used as for patients with $\mathrm{CF}^{36}$. Additionally, SCD primarily affects African Americans, Hispanic Americans, and other underrepresented minorities who already suffer from health care disparities ${ }^{35}$. Given the burden of pulmonary complications in this chronic disease, adding a pulmonologist to the SCD care model could further enrich the multidisciplinary experience and improve health outcomes for this traditionally disadvantaged population. In fact, comprehensive care involving multiple medical subspecialties has been shown to reduce hospitalizations for adults with SCD at a university hospital in London ${ }^{37}$.

There are several limitations to this study. Due to the retrospective design, the study start and end times for the patients were set somewhat arbitrarily. Randomized control trials are needed to more directly compare outcomes between children who received pulmonary care and those who did not. Additionally, this was a single center study which took place over a limited number of years. Although the majority of SCD patients at $\mathrm{CHOP}$ receive all their care at this institution, it is possible that some patients had hospital visits at another institution which were not captured. Larger multicenter studies would help more accurately estimate hospitalization rates for this population.

In conclusion, this study supports the role of the pulmonologist in the multidisciplinary care of children with SCD and asthma. Evaluation and management of children with SCD and asthma by a pulmonologist was shown to improve outcomes, as demonstrated by more standardized asthma care and decreased rates of ED visits and hospitalizations for asthma exacerbation and ACS. Further studies are needed to explore the effects of specific therapeutic interventions and establish evidence-based guidelines to improve long term pulmonary outcomes in children with SCD.

\section{References}

1. National Heart, Lung, and Blood Institute. Evidence-based management of sickle cell disease: Expert panel report, 2014. U.S. Department of Health and Human Services. 2014 Sep:1-161.

2. Knight-Madden JM. Asthma in children with sickle cell disease and its association with acute chest syndrome. Thorax. 2005;60(3):206-210. doi:10.1136/thx.2004.029165

3. Ruhl AP, Sadreameli SC, Allen JL, Bennett DP, Campbell AD, Coates TD, Diallo DA, Field JJ, Fiorino EK, Gladwin MT, et al. Identifying clinical and research priorities in sickle cell lung disease: An official American Thoracic Society workshop report. Annals of the American Thoracic Society. 2019;16(9):e17-e32. doi:10.1513/AnnalsATS.201906-433ST

4. Platt OS, Brambilla DJ, Rosse WF, Milner PF, Castro O, Steinberg MH, Klug PP. Mortality in sickle cell disease: Life expectancy and risk factors for early death. The New England Journal of Medicine. 1994;330(23):1639-1644. doi:10.1056/NEJM199406093302303

5. Koumbourlis AC, Zar HJ, Hurlet-Jensen A, Goldberg MR. Prevalence and reversibility of lower airway obstruction in children with sickle cell disease. The Journal of Pediatrics. 2001;138(2):188-192. doi:10.1067/mpd.2001.111824

6. Field JJ, DeBaun MR, Yan Y, Strunk RC. Growth of lung function in children with sickle cell anemia: Lung Growth in Sickle Cell Disease. Pediatric Pulmonology. 2008;43(11):1061-1066. doi:10.1002/ppul.20883 
7. Ivankovich DT, Braga JAP, Lanza F de C, Solé D, Wandalsen GF. Lung function in infants with sickle cell anemia. The Journal of Pediatrics. 2019;207:252-254. doi:10.1016/j.jpeds.2018.11.036

8. Leong MA, Dampier C, Varlotta L, Allen JL. Airway hyperreactivity in children with sickle cell disease. The Journal of Pediatrics. 1997;131(2):278-283. doi:10.1016/S0022-3476(97)70166-5

9. Andemariam B, Adami AJ, Singh A, McNamara JT, Secor ER, Guernsey LA, Thrall RS. The sickle cell mouse lung: proinflammatory and primed for allergic inflammation. Translational Research. 2015;166(3):254268. doi:10.1016/j.trsl.2015.03.001

10. Nandedkar SD, Feroah TR, Hutchins W, Weihrauch D, Konduri KS, Wang J, Strunk RC, DeBaun MR, Hillery CA, Pritchard KA. Histopathology of experimentally induced asthma in a murine model of sickle cell disease. Blood. 2008;112(6):2529-2538. doi:10.1182/blood-2008-01-132506

11. Glassberg JA, Strunk R, DeBaun MR. Wheezing in children with sickle cell disease: Current Opinion in Pediatrics. 2014;26(1):9-18. doi:10.1097/MOP.0000000000000045

12. Morris CR. Asthma management: Reinventing the wheel in sickle cell disease. American Journal of Hematology. 2009;84(4):234-241. doi:10.1002/ajh.21359

13. Newaskar M, Hardy KA, Morris CR. Asthma in sickle cell disease. The Scientific World Journal. 2011;11:1138-1152. doi:10.1100/tsw.2011.105

14. DeBaun MR, Strunk RC. The intersection between asthma and acute chest syndrome in children with sickle-cell anaemia. The Lancet. 2016;387(10037):2545-2553. doi:10.1016/S0140-6736(16)00145-8

15. Nordness ME, Lynn J, Zacharisen MC, Scott PJ, Kelly KJ. Asthma is a risk factor for acute chest syndrome and cerebral vascular accidents in children with sickle cell disease. Clinical and Molecular Allergy. $2005 ; 3(1): 2$. doi:10.1186/1476-7961-3-2

16. Strunk RC, Cohen RT, Cooper BP, Rodeghier M, Kirkham FJ, Warner JO, Stocks J, Kirkby J, Roberts I, Rosen CL, et al. Wheezing symptoms and parental asthma are associated with a physician diagnosis of asthma in children with sickle cell anemia. The Journal of Pediatrics. 2014;164(4):821-826.e1. doi:10.1016/j.jpeds.2013.11.034

17. Boyd JH, Macklin EA, Strunk RC, DeBaun MR. Asthma is associated with acute chest syndrome and pain in children with sickle cell anemia. Blood. 2006;108(9):2923-2927. doi:10.1182/blood-2006-01-011072

18. Glassberg JA, Wang J, Cohen R, Richardson LD, DeBaun MR. Risk factors for increased ED utilization in a multinational cohort of children with sickle cell disease: ED use by sickle cell patients. Academic Emergency Medicine. 2012;19(6):664-672. doi:10.1111/j.1553-2712.2012.01364.x

19. Boyd JH, Macklin EA, Strunk RC, DeBaun MR. Asthma is associated with Increased mortality in individuals with sickle cell anemia. Haematologica. 2007;92(8):1115-1118. doi:10.3324/haematol.11213

20. Anim SO, Strunk RC, DeBaun MR. Asthma morbidity and treatment in children with sickle cell disease. Expert Review of Respiratory Medicine. 2011;5(5):635-645. doi:10.1586/ers.11.64

21. Price D, Bjermer L, Bergin D, Martinez R. Asthma referrals: a key component of asthma management that needs to be addressed. Journal of Asthma and Allergy. 2017;Volume10:209-223. doi:10.2147/JAA.S134300

22. Hoover NG, Callahan CW. Reduced asthma morbidity following pediatric pulmonary consultation. Pediatric Pulmonology. 1999;28(5):383. doi:10.1002/(SICI)1099-0496(199911)28:5<383::AID-PPUL12>3.0.CO $2-\mathrm{Z}$

23. McClain BL, Ivy ZK, Bryant V, Rodeghier M, DeBaun MR. Improved guideline adherence with integrated sickle cell disease and asthma care. American Journal of Preventive Medicine. 2016;51(1):S62-S68. doi:10.1016/j.amepre.2016.03.003 
24. Sylvester KP, Patey RA, Milligan P, Rafferty GF, Broughton S, Rees D, Thein SL, Greenough A. Impact of acute chest syndrome on lung function of children with sickle cell disease. The Journal of Pediatrics. 2006;149(1):17-22. doi:10.1016/j.jpeds.2005.12.059

25. Boyd JH, DeBaun MR, Morgan WJ, Mao J, Strunk RC. Lower airway obstruction is associated with increased morbidity in children with sickle cell disease. Pediatric Pulmonology. 2009;44(3):290-296. doi:10.1002/ppul.20998

26. Cohen RT, Strunk RC, Rodeghier M, Rosen CL, Kirkham FJ, Kirkby J, DeBaun MR. Pattern of lung function is not associated with prior or future morbidity in children with sickle cell anemia. Annals of the American Thoracic Society. 2016;13(8):1314-1323. doi:10.1513/AnnalsATS.201510-706OC

27. Pahl K, Mullen CA. Acute chest syndrome in sickle cell disease: Effect of genotype and asthma. Experimental Biology and Medicine. 2016;241(7):745-758. doi:10.1177/1535370216636720

28. Kaleyias J, Mostofi N, Grant M, Coleman C, Luck L, Dampier C, Kothare SV. Severity of obstructive sleep apnea in children with sickle cell disease. Journal of Pediatric Hematology/Oncology. 2008;30(9):659665. doi:10.1097/MPH.0b013e31817eb7ef

29. Miller AC, Gladwin MT. Pulmonary complications of sickle cell disease. American Journal of Respiratory and Critical Care Medicine. 2012;185(11):1154-1165. doi:10.1164/rccm.201111-2082CI

30. Knight-Madden JM, Hambleton IR. Inhaled bronchodilators for acute chest syndrome in people with sickle cell disease Cochrane Cystic Fibrosis and Genetic Disorders Group, editor. Cochrane Database of Systematic Reviews. 2016 Sep 27. doi:10.1002/14651858.CD003733.pub4

31. Leonard A, Godiwala N, Herrera N, McCarter R, Sharron M, Meier ER. Early initiation of inhaled corticosteroids does not decrease acute chest syndrome morbidity in pediatric patients with sickle cell disease. Blood Cells, Molecules, and Diseases. 2018;71:55-62. doi:10.1016/j.bcmd.2018.03.001

32. Sobota A, Graham DA, Heeney MM, Neufeld EJ. Corticosteroids for acute chest syndrome in children with sickle cell disease: Variation in use and association with length of stay and readmission. American Journal of Hematology. 2010;85(1):24-28. doi:10.1002/ajh.21565

33. Strouse JJ, Takemoto CM, Keefer JR, Kato GJ, Casella JF. Corticosteroids and increased risk of readmission after acute chest syndrome in children with sickle cell disease: Acute Chest Syndrome in Sickle Cell Disease. Pediatric Blood \& Cancer. 2008;50(5):1006-1012. doi:10.1002/pbc.21336

34. Smith LA. Sickle Cell Disease: A Question of Equity and Quality. PEDIATRICS. 2006;117(5):1763-1770. doi:10.1542/peds.2005-1611

35. King AA, Baumann AA. Sickle cell disease and implementation science: A partnership to accelerate advances. Pediatric Blood \& Cancer. 2017;64(11):e26649. doi:https://doi.org/10.1002/pbc.26649

36. Grosse SD, Schechter MS, Kulkarni R, Lloyd-Puryear MA, Strickland B, Trevathan E. Models of Comprehensive Multidisciplinary Care for Individuals in the United States With Genetic Disorders. PEDIATRICS. 2009;123(1):407-412. doi:10.1542/peds.2007-2875

37. Okpala I, Thomas V, Westerdale N, Jegede T, Raj K, Daley S, Costello-Binger H, Mullen J, RochesterPeart C, Helps S, et al. The comprehensive care of sickle cell disease. European Journal of Haematology. 2002;68(3):157-162. doi:https://doi.org/10.1034/j.1600-0609.2002.01523.x

\section{Hosted file}

Tables final.docx available at https://authorea.com/users/416605/articles/524083-benefit-ofpulmonary-subspecialty-care-for-children-with-sickle-cell-disease-and-asthma 


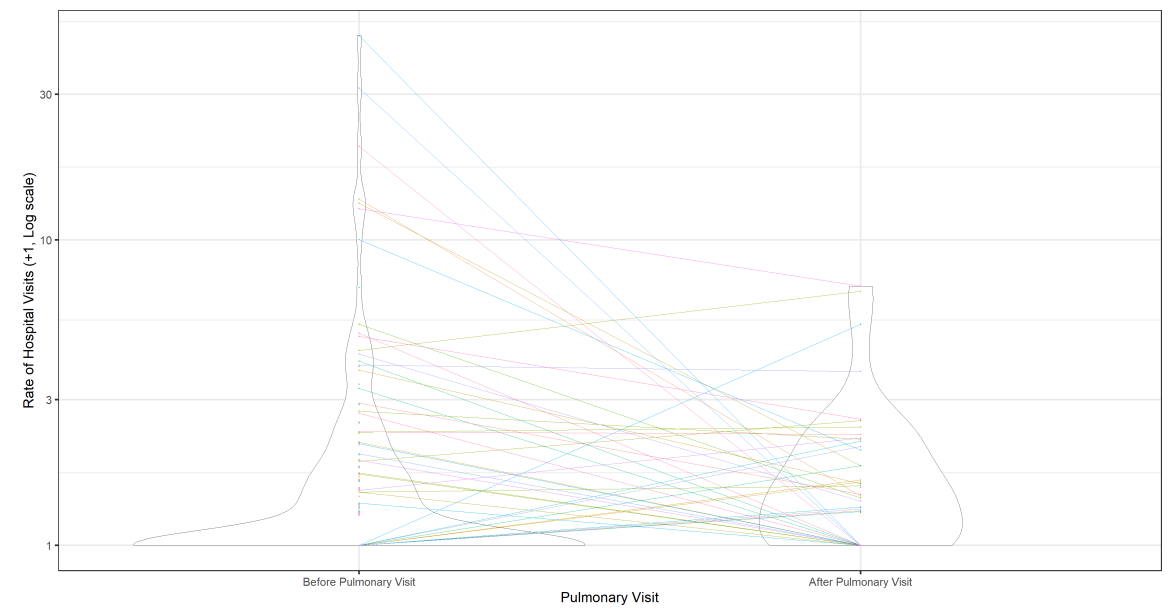

